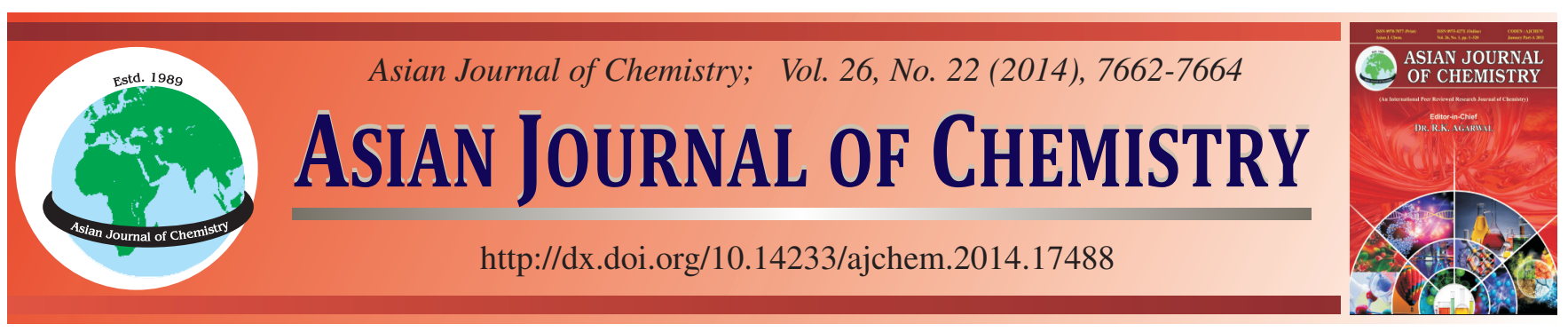

\title{
Synthesis, Crystal Structure and Characterization of Zn(II) Complex Constructed from 2,2'-Bisbenzimidazole
}

Yan Yang ${ }^{1, *}$, Chang-Gui Li ${ }^{2}$ Xu-Jian Luo ${ }^{1}$, Zhi-Hui Luo ${ }^{1}$, Rong-Jun Liu ${ }^{1}$, Yue-Xiu Jiang ${ }^{2}$ and Wei-Jiang Liang ${ }^{1, *}$

${ }^{1}$ School of Chemistry and Material, Yulin Normal University, Yulin 537000, Guangxi Province, P.R. China

${ }^{2}$ School of Chemistry and Chemical Engineering, Guangxi University, Nanning 530004, Guangxi Province, P.R. China

*Corresponding authors: Tel/Fax: +86 775 2622315; E-mail: yy135175@163.com; liang2179@163.com

\begin{abstract}
A new complex associated with $\mathrm{Zn}$ ion, $\left[\mathrm{Zn}\left(\mathrm{OH}-\mathrm{H}_{2} \mathrm{Bdc}\right)(\mathrm{Bibim})\right]_{\mathrm{n}}(\mathbf{1}),\left(\mathrm{OH}-\mathrm{H}_{2} \mathrm{Bdc}=5\right.$-hydroxyisophthalic acid, Bibim = 2,2'bisbenzimidazole) has been synthesized under hydrothermal conditions and characterized by elemental analysis, FT-IR spectroscopy, TG/DTG and fluorescence spectra. X-ray crystallographic studies reveal that complex $\left[\mathrm{Zn}\left(\mathrm{OH}-\mathrm{H}_{2} \mathrm{Bdc}\right)(\mathrm{Bibim})\right]_{\mathrm{n}}(\mathbf{1})$ crystallizes in a monoclinic space group $\mathrm{P} 2_{1} / \mathrm{n}$, each $\mathrm{Zn}$ atom is five-coordinated and adopts a distorted square-pyramidal coordination environment. There exists a 2-D supramolecular network linked by hydrogen bonds and face-to-face $\pi$ - $\pi$ interactions between adjacent benzene rings $(3.768 \AA)$ and imidazole rings $(3.742 \AA)$. A three-dimensional supramolecular network is further constructed through $\pi-\pi$ stacking and hydrogen-bonding interactions between the helices. The TG/DTG shows that it collapsed with total loss of 84.8 (calc. $86.3 \%$ ). Blue fluorescent emission of the complex was determined at $506 \mathrm{~nm}$ in the solid state.
\end{abstract}

Keywords: Zinc(II) complex, 2,2'-Bisbenzimidazole, Crystal structure, Fluorescent property.

\section{INTRODUCTION}

The design and synthesis of novel coordination polymers have received remarkable attention not only due to their intriguing variety of structures and topologies, but also to their potential applications in many fields such as ion exchange, catalysis and for the development of optical, electronic and magnetic devices ${ }^{1-4}$. However, it is still a challenge to construct coordination polymer, which may be affected by many factors, such as metal ions, organic ligands, metal-to-ligand ratios, reaction temperature, solvents, and counter ions ${ }^{5-7}$. Among these factors, metal ions and organic ligands play crucial roles in synthesizing new coordination polymer. So various multicarboxylate ligands have been used to produce coordination polymer, due to their versatile bridge modes and excellent coordination capacities. As a rigid and versatile bridging ligand, 5-hydroxyisophthalic acid has been extensively studied for designing new coordination polymer because its two carboxylic groups can bond with metal centers and the hydroxyl group, an electron-with drawing group coexisting in isophthalic acid, can not only act as a hydrogen bond acceptor, can but also take on some spatial effects ${ }^{8,9}$. On the other hand, benzimidazole derivatives contain multiple nitrogen-donor sites with the possibility of reversible protonation and deprotonation properties and can be coordinated to a transition metal in various forms, which has been utilized in coordination polymer for the preparation of different networks ${ }^{10-12}$. Herein, we report the synthesis, X-ray crystal structure, thermal and optical spectral properties of a novel zinc(II) complex $\left[\mathrm{Zn}\left(\mathrm{OH}-\mathrm{H}_{2} \mathrm{Bdc}\right)(\mathrm{Bibim})\right]_{\mathrm{n}}(\mathbf{1})$.

\section{EXPERIMENTAL}

All solutions and chemicals were commercial reagents and used without further purification. Elemental analysis was carried out on a PE $1700 \mathrm{CHN}$ auto elemental analyzer. The solid infrared spectra (IR) were obtained from a Bruker IFS66V vacuumtype FT-IR spectrophotometer using $\mathrm{KBr}$ pellets. Thermogravimetry (TG) analysis was measured on a Perkin Elmer TG/DTA 6300 thermal analyzer under flowing $\mathrm{N}_{2}$ at a heating rate of $10{ }^{\circ} \mathrm{C} \mathrm{min}^{-1}$. Fluorescence measurements were performed on a Model RF-5 spectrofluorimeter. The crystal structure was determined using a Bruker APEX area-detector diffractometer and SHELXL crystallographic software.

Synthesis of $\left[\mathrm{Zn}\left(\mathrm{OH}-\mathrm{H}_{2} \mathrm{Bdc}\right)(\mathrm{Bibim})\right]_{\mathbf{n}}(\mathbf{1})$ : For the hydrothermal reaction of $\mathrm{Zn}\left(\mathrm{NO}_{3}\right)_{2} \cdot 6 \mathrm{H}_{2} \mathrm{O}(0.5 \mathrm{mmol})$, bisbenzimidazole $(0.25 \mathrm{mmol}), 5$-hydroxyisophthalic acid $(0.25 \mathrm{mmol})$, and water $(15 \mathrm{~mL})$, the mixture was stirred for $10 \mathrm{~min}$ in air, then transferred and sealed into a $23 \mathrm{~mL}$ Teflon reactor, which was heated at $100{ }^{\circ} \mathrm{C}$ for two days and then cooled to room temperature. Colorless crystals were obtained (yield $48 \%$ 
based on $\mathrm{Zn}$ ), filtered off, washed with distilled water and dried in air. Elemental analysis (\%): Anal. Calcd. C, 55.08; H, 2.94; $\mathrm{N}, 11.68$; found C, 54.96; H, 3.11; N, 11.87. IR (KBr, $v_{\max }$, $\left.\mathrm{cm}^{-1}\right): 3379,1607,1588,1539,1395,1349,1259,1149,1116$, 1001, 976, 921, 776, 744.

X-ray crystallography: The diffraction data were collected on a Bruker Smart Apex CZN diffractometer with graphite-monochromated $\operatorname{MoK}_{\alpha}$ radiation $(\lambda=0.71073 \AA)$ at 296 K. Absorption correction was applied by SADABS ${ }^{13}$. The structure was solved by Direct Methods and refined with fullmatrix least-squares technique using SHELXTL ${ }^{14}$. All nonhydrogen atoms were refined with anisotropic displacement parameters. Complete crystallographic data (CCDC 827564) has been deposited at The Cambridge Crystallographic Data Centre and may be obtained free of charge via www.cznc.cam.ac.uk/data-request/cif. The crystal data, details on the data collection and refinement are summarized in Table-1, and selected bond lengths and angles are presented in Table- 2 . The hydrogen bond lengths (nm) and bond angles (deg) are listed in Table-3. Figs. 1-3 illustrate the structure of $\mathbf{1}$.

\begin{tabular}{|c|c|}
\hline \multicolumn{2}{|c|}{$\begin{array}{c}\text { TABLE-1 } \\
\text { CRYSTAL DATA AND STRUCTURE } \\
\text { REFINEMENT FOR COMPLEX } 1\end{array}$} \\
\hline Empirical formula & $\mathrm{C}_{22} \mathrm{H}_{14} \mathrm{~N}_{4} \mathrm{O}_{5} \mathrm{Zn}$ \\
\hline Formula weight & 479.77 \\
\hline Temp. (K) & $296(2)$ \\
\hline Crystal system & Monoclinic \\
\hline Space group & $\mathrm{P} 2_{1} / \mathrm{n}$ \\
\hline$a(\AA)$ & $7.4211(14)$ \\
\hline $\mathrm{b}(\AA)$ & $12.278(2)$ \\
\hline c $(\AA)$ & $21.550(4)$ \\
\hline$\alpha\left(^{\circ}\right)$ & 90 \\
\hline$\beta\left({ }^{\circ}\right)$ & $95.515(3)$ \\
\hline$\gamma\left({ }^{\circ}\right)$ & 90 \\
\hline $\mathrm{V}\left(\AA^{3}\right)$ & $1954.5(6)$ \\
\hline Cryst size (mm) & $0.18 \times 0.20 \times 0.22$ \\
\hline $\mathrm{Z}$ & 4 \\
\hline $\mathrm{D}_{\mathrm{c}}\left(\mathrm{g} \mathrm{cm}^{-3}\right)$ & 1.630 \\
\hline$\mu(\mathrm{cm})$ & 13.0 \\
\hline $\mathrm{F}(000)$ & 976 \\
\hline Limiting indices & $\begin{array}{c}-7 \leq \mathrm{h} \leq 8 ;-13 \leq \mathrm{k} \leq 14 \\
-23 \leq 1 \leq 25\end{array}$ \\
\hline Reflections & 10253 \\
\hline Independent & 3436 \\
\hline Observed data & 2614 \\
\hline Npar & 290 \\
\hline $\mathrm{R}_{\mathrm{int}}$ & 0.070 \\
\hline GOF & 1.03 \\
\hline $\left.\mathrm{R}_{1}^{\mathrm{a}}(\mathrm{I}>2 \sigma \mathrm{I})\right)$ & 0.0392 \\
\hline $\mathrm{wR}_{2}^{\mathrm{a}}$ (all data) & 0.1139 \\
\hline Max/min electron density $\left(e \AA^{-3}\right)$ & $0.58 /-0.46$ \\
\hline Theta range $\left(^{\circ}\right)$ & $1.9,25.0$ \\
\hline
\end{tabular}

\section{RESULTS AND DISCUSSION}

Single-crystal X-ray crystallographic studies reveal that complex 1 crystallizes in a monoclinic space group $\mathrm{P} 2_{1} / \mathrm{n}$, which is made up of one $\mathrm{Zn}(\mathrm{II})$, one Bibim and one Bdc (Bdc =5-hydroxyisophthalate) molecule. Each Zn atom is five-coordinated and adopts a distorted square-pyramidal coordination

\begin{tabular}{|c|c|c|c|}
\hline \multicolumn{4}{|c|}{$\begin{array}{c}\text { TABLE- } 2 \\
\text { SELECTED BOND LENGTHS }(\AA) \text { AND } \\
\text { BOND ANGLES }\left({ }^{\circ}\right) \text { FOR COMPLEX } 1\end{array}$} \\
\hline $\mathrm{Zn1-O1}$ & $2.264(3)$ & Zn1-N1 & $2.146(3)$ \\
\hline $\mathrm{Zn} 1-\mathrm{O} 2$ & $2.068(2)$ & Zn1-N3 & $2.046(3)$ \\
\hline $\mathrm{Zn} 1-\mathrm{O} 3$ & $1.984(3)$ & - & - \\
\hline $\mathrm{O} 1-\mathrm{Zn} 1-\mathrm{O} 2$ & $60.59(9)$ & $\mathrm{O} 2-\mathrm{Zn} 1-\mathrm{C} 15$ & $30.76(11)$ \\
\hline O1-Zn1-O3 & $89.64(9)$ & O3-Zn1-N1 & $96.33(10)$ \\
\hline O1-Zn1-N1 & $171.05(10)$ & $\mathrm{O} 3-\mathrm{Zn} 1-\mathrm{N} 3$ & $144.45(12)$ \\
\hline O1-Zn1-N3 & $98.36(9)$ & O3-Zn1-C15 & $93.38(10)$ \\
\hline O1-Zn1-C15 & $30.09(10)$ & N1-Zn1-N3 & $80.62(10)$ \\
\hline O2-Zn1-O3 & $101.40(11)$ & N1-Zn1-C15 & $142.23(12)$ \\
\hline $\mathrm{O} 2-\mathrm{Zn} 1-\mathrm{N} 1$ & $111.48(11)$ & N3-Zn1-C15 & $110.73(10)$ \\
\hline O2-Zn1-N3 & $112.74(11)$ & - & - \\
\hline
\end{tabular}

environment (Fig. 1). Two $\mathrm{N}$ atoms are from one Bibim ligand and three $\mathrm{O}$ atoms from two $\mathrm{Bdc}$ ligands. The $\mathrm{Zn}-\mathrm{O}$ distances are in the range of 1.984(3)-2.264(3) $\AA$, and the $\mathrm{Zn}-\mathrm{N}$ distances are in the range of 2.046(3)-2.146(3) $\AA$, respectively. In the structure, the Bdc ligand adopts $\mu_{3}$-bridging coordination fashion : one carboxylate group adopts chelating/bridging mono-bidentate coordination mode to chelate the $\mathrm{Zn}$ (II) centers, and another carboxylate group acts as a mono-dentate ligand, while Bibim ligand acts as a chelating ligand. Each pair of adjacent $\mathrm{Zn}$ atoms is bridged by Bdc ligands to form a helical chain running along a direction with a long pitch of $7.36 \AA$. These chains are decorated with Bibim ligands, positioning alternately on two sides and pointing outwards, as depicted in Fig. 2. The adjacent helices are alternately packed through hydrogen bonds involving Bdc and Bibim ligands (Table-3) and face-to-face $\pi-\pi$ interactions between adjacent benzene rings $(3.768 \AA)$ and imidazole rings $(3.742 \AA)$ to sustain the 2D-assembly extended in the ab plane, as illustrated in Fig. 3. A three-dimensional supramolecular network is further constructed through $\pi-\pi$ stacking and hydrogen-bonding interactions between the helices.

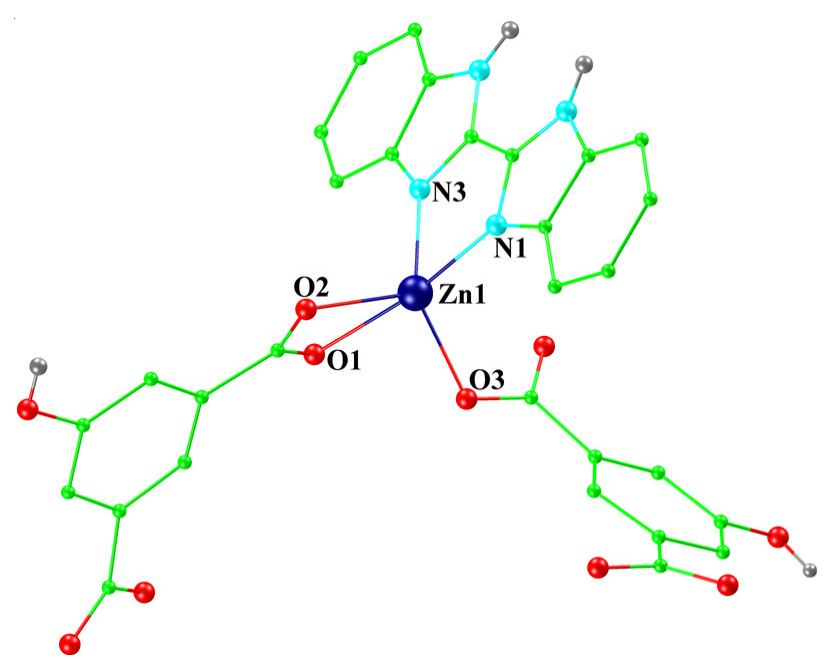

Fig. 1. Coordination environment of $\mathrm{Zn}$ (II) ion in $\mathbf{1}$

Thermogravimetric analysis was carried out to examine the thermal stability of complex $\mathbf{1}$ (Fig. 4). The crushed singlecrystal sample was heated up to $1000{ }^{\circ} \mathrm{C}$ in $\mathrm{N}_{2}$ at a heating rate of $10{ }^{\circ} \mathrm{C} \mathrm{min}{ }^{-1}$. The TG and DTG curves for 1 shown that it collapsed with total loss of $84.8 \%$ (calc. $86.3 \%$ ), consistent with the pyrolysis of mixed-ligands. The pyrolysis of mixed- 
TABLE-3

DISTANCES $(\AA)$ AND ANGLES $\left({ }^{\circ}\right)$ OF HYDROGEN BONDS FOR COMPLEX 1

\begin{tabular}{cccccc}
\hline $\mathrm{D}-\mathrm{H} \cdots \mathrm{A}$ & $\mathrm{d}(\mathrm{D}-\mathrm{H})$ & $\mathrm{d}(\mathrm{H} \cdots \mathrm{A})$ & $\mathrm{d}(\mathrm{D} \cdots \mathrm{A})$ & \multicolumn{1}{c}{$L(\mathrm{D}-\mathrm{H} \cdots \mathrm{A})$} & \\
\hline $\mathrm{N} 2-\mathrm{H} 2 \cdots \mathrm{O} 2$ & 0.8607 & 2.2765 & $2.851(4)$ & 124.26 & $-\mathrm{x}, 2-\mathrm{y}, 1-\mathrm{z}$ \\
$\mathrm{N} 2-\mathrm{H} 2 \cdots \mathrm{O} 4$ & 0.8607 & 2.5409 & $3.251(4)$ & 140.46 & $1 / 2+\mathrm{x}, 3 / 2-\mathrm{y},-1 / 2+\mathrm{z}$ \\
$\mathrm{N} 4-\mathrm{H} 4 \cdots \mathrm{O} 4$ & 0.8595 & 1.9153 & $2.709(4)$ & 152.91 & $1 / 2+\mathrm{x}, 3 / 2-\mathrm{y},-1 / 2+\mathrm{z}$ \\
$\mathrm{O} 5-\mathrm{H} 5 \cdots \mathrm{O} 1$ & 0.8209 & 1.9798 & $2.707(4)$ & 147.30 & $-1+\mathrm{x}, \mathrm{y}, \mathrm{z}$ \\
$\mathrm{C} 7-\mathrm{H} 7 \cdots \mathrm{O} 5$ & 0.9308 & 2.4592 & $3.358(5)$ & 162.21 & $1 / 2-\mathrm{x}, 1 / 2+\mathrm{y}, 3 / 2-\mathrm{z}$ \\
$\mathrm{C} 22-\mathrm{H} 22 \cdots \mathrm{O} 5$ & 0.9297 & 2.4343 & $3.326(4)$ & 160.72 & $1+\mathrm{x}, \mathrm{y}, \mathrm{z}$ \\
\hline
\end{tabular}

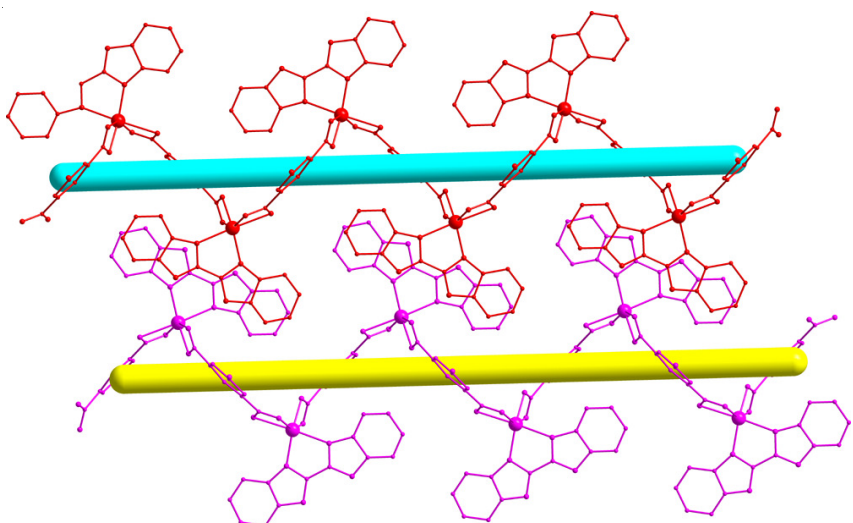

Fig. 2. 1D helical chain of $\mathbf{1}$

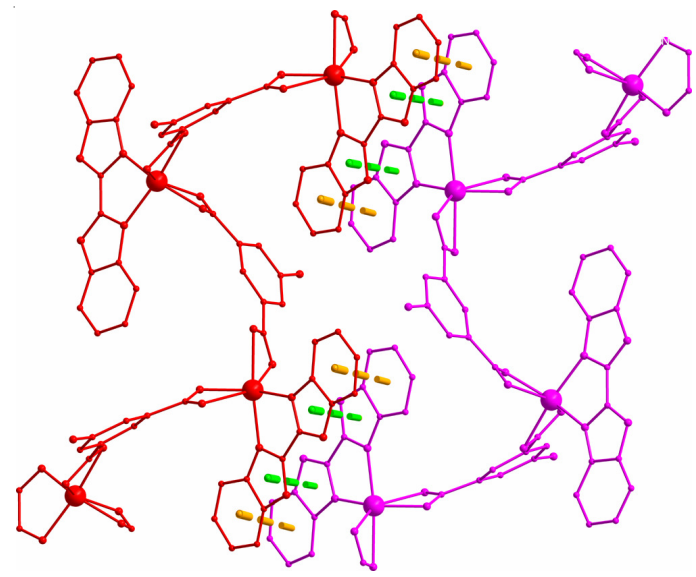

Fig. 3. $\pi-\pi$ interactions from two nearest $1 \mathrm{D}$ chains of $\mathbf{1}$

ligands occurred in the temperature range $100-800{ }^{\circ} \mathrm{C}$. The above different thermal behaviours may attribute to their structural features, the presence of a number of coordination bond of carboxylate ligands and the chelating effect of five-membered rings, which change the bite angles for chelating, resulting in the formation of a rich variety of polymeric structures.

The emission spectrum of complex $\mathbf{1}$ in the solid state is investigated at room temperature. Excitation at $365 \mathrm{~nm}$ leads to strong blue-fluorescent emission band at $506 \mathrm{~nm}$ for $\mathbf{1}$. The emission is neither metal-to-ligand charge transfer (MLCT) nor ligand-to-metal transfer (LMCT) in nature since the $\mathrm{Zn}$ (II) ions are difficult to oxidize or to reduce due to their $d^{10}$ configuration. For the free $\mathrm{H}_{2} \mathrm{Bdc}$ ligand, the emission band at 387 $\mathrm{nm}\left(\lambda_{\mathrm{ex}}=351 \mathrm{~nm}\right)$ can be assigned to $\pi-\pi *$ transition. In addition, for the free Bibim, the main emission band at 498$\mathrm{nm}\left(\lambda_{\mathrm{ex}}=490 \mathrm{~nm}\right)$, is assigned to the intraligand $\pi-\pi *$ transition. Therefore, we assign the emission described above for $\mathbf{1}$ to LLCT (ligand-ligand charge transfer) excited states.

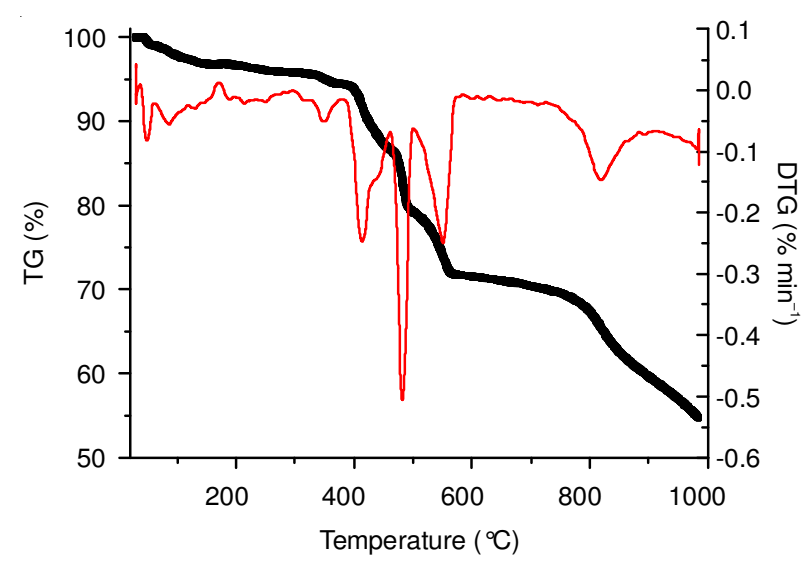

Fig. 4 TG-DTG curve of $\mathrm{Zn}(\mathrm{II})$ complex

In summary, a zinc(II) coordination polymer based on $\mathrm{OH}-\mathrm{H}_{2} \mathrm{Bdc}$ and Bibim was hydrothermally synthesized and exhibits intense blue photoluminescent emission. The TG/DTG shows that it collapsed with total loss of $84.8 \%$. The complex is promising as a blue-light emitting material for its high thermal stability and the insolubility in common solvents.

\section{ACKNOWLEDGEMENTS}

This work was financially supported by the National Natural Science Foundation of China (No. 21341005).

\section{REFERENCES}

1. B.L. Chen, S.C. Xiang and G.D. Qian, Acc. Chem. Res., 43, 1115 (2010).

2. O.K. Farha, C.D. Malliakas, M.G. Kanatzidis and J.T. Hupp, J. Am. Chem. Soc., 132, 950 (2010).

3. J.R. Li, R.J. Kuppler and H.C. Zhou, Chem. Soc. Rev., 38, 1477 (2009).

4. J.P. Zhang and X.M. Chen, J. Am. Chem. Soc., 131, 5516 (2009).

5. J.Q. Liu, Y.Y. Wang, Y.N. Zhang, P. Liu, Q.Z. Shi and S.R. Batten, Eur. J. Inorg. Chem., 147 (2009).

6. J.L. Du, T.L. Hu, J.R. Li, S.M. Zhang and X.H. Bu, Eur. J. Inorg. Chem., 1059 (2008).

7. F.Y. Cui, K.L. Huang, Y.Q. Xu, Z.G. Han, X. Liu, Y.N. Chi and C.W. $\mathrm{Hu}$, CrystEngComm, 11, 2757 (2009).

8. L. Liu, H. Zhang, X.G. Meng, J. Yin, D.F. Li and C.L. Liu, Biomaterials, 31, 1380 (2010).

9. H.K. Liu and P.J. Sadler, Acc. Chem. Res., 44, 349 (2011).

10. Y. Yang, M.X. Tan, X. Li, X.M. Chen, G.Q. Chen, R.H. Qin and W.G. Duan, J. Mol. Struct., 975, 372 (2010).

11. Y. Yang, L.T. Yan, W.G. Duan, X. Li and R.H. Qin, Supramol. Chem., 24, 707 (2012).

12. Y. Yang, L.T. Yan, X.J. Luo, R.H. Qin and W.G. Duan, Supramol. Chem., 24, 810 (2012).

13. G.M. Sheldrick, SHELXS-97, Program for Crystal Structure Determination, University of Göttingen, Göttingen, Germany (1997).

14. G.M. Sheldrick, SHELXL-97, Program for Crystal Structure Refinement, University of Göttingen, Göttingen, Germany (1997). 